\title{
The Dynamics of Dendrimers by NMR Relaxation: Interpretation Pit- falls
}

\author{
Luiz F. Pinto, ${ }^{\dagger}$ Juan Correa, ${ }^{\dagger}$ Manuel Martin-Pastor, ${ }^{\ddagger}$ Ricardo Riguera, ${ }^{*,+}$ and Eduardo Fernandez-Megia ${ }^{*, \dagger}$ \\ ${ }^{\dagger}$ Department of Organic Chemistry and Center for Research in Biological Chemistry and Molecular Materials (CIQUS), University of \\ Santiago de Compostela, Jenaro de la Fuente s/n, 15782 Santiago de Compostela, Spain. ${ }^{\ddagger}$ Unidade de Resonancia Magnética, \\ RIAIDT, CACTUS, University of Santiago de Compostela, 15782 Santiago de Compostela, Spain.
}

\begin{abstract}
NMR is a powerful tool to study the dynamics of dendrimers. By analogy to linear polymers, shorter $T_{1}$ relaxation times have been traditionally associated to less mobile nuclei and hence, dendrimers described with reduced local motions either at the core or the periphery. Herein we report a NMR relaxation study $\left[{ }^{1} \mathrm{H}\right.$ and ${ }^{13} \mathrm{C} T_{1}, T_{2} ;{ }^{13} \mathrm{C}\left\{{ }^{1} \mathrm{H}\right\} \mathrm{NOE}$; various fields and temperatures] which reveals profound differences between the relaxation behavior of dendrimers and linear polymers. Dendrimers show slower dynamics at internal layers and on increasing generation, and may display internal nuclei in the slow motional regime with larger $T_{1}$ values than the periphery. In contrast to the relaxation properties of linear polymers, these $T_{1}$ increments should not be interpreted as resulting from faster dynamics. Only the recording of $T_{1}$ data at various temperatures (alternatively, $T_{2}$ or NOE at one temperature) ensures the correct interpretation of dendrimer dynamics.
\end{abstract}

\section{INTRODUCTION}

Dendrimers constitute an exciting opportunity for scientists to create globular, highly branched, and perfectly monodisperse macromolecules with applications in numerous fields. ${ }^{1}$ The characteristic architecture of dendrimers by generations (G1, G2, G3...) determines their physico-chemical properties and function, which has attracted much attention to their density distribution and dynamics. Conflicting theoretical models initially proposed by de Gennes/Hervet (dense-shell), ${ }^{2}$ and Lescanec/Muthukumar (dense-core) ${ }^{3}$ described segmental density profiles with global maxima at the periphery and core, respectively. More recently, a consensus has emerged with the majority of theoretical models, computer simulations, and experimental studies (small angle neutron and X-ray scattering) pointing to a density distribution close to that predicted by Lescanec and Muthukumar. ${ }^{4}$

Nuclear magnetic resonance (NMR) is a powerful tool to study the dynamics of macromolecules at atomic level..$^{5}$ Information is usually extracted by measuring longitudinal $\left(T_{1}\right)$ and transverse $\left(T_{2}\right)$ relaxation times, and nuclear Overhauser effect (NOE) ${ }^{6,7}$ It is especially suited for the analysis of dendrimers since their repetitive nature offers the opportunity to probe different layers and G. Quantitative modeling of dendrimer dynamics is feasible from ${ }^{13} \mathrm{C}$ relaxation, but lengthy experiments and the necessity of recording various relaxation parameters (typically $T_{1}, T_{2}$, and NOE) at different magnetic fields limit such approach. ${ }^{8}$ Conversely, a great deal of information has been extracted by qualitative interpretation of ${ }^{1} \mathrm{H}$ and/or ${ }^{13} \mathrm{C}$ relaxation. ${ }^{9,10,11,12,13}$ These studies have, nevertheless, afforded conflicting results on the relative dynamics between the dendritic core and the periphery which, in our opinion, might stem from interpretation pitfalls of the relaxation data.

With the aim of throwing light on this controversy, one should bear in mind the theoretical variation of $T_{1}$ and $T_{2}$ with the correlation time $(\tau)$ and their experimental dependence on the molecular weight (MW). Thus, for a spin in a simple isotropic and rigid model, $T_{2}$ decreases monotonically with $\tau$, whereas $T_{1}$ decreases down to a minimum (where $\omega^{2} \tau^{2} \sim 1.12$, being $\omega$ the Larmor frequency) to increase afterwards. This renders two possible $\tau$ for a given $T_{1}$ value, at the fast $\left(\omega^{2} \tau^{2}<1.12\right)$ and slow $\left(\omega^{2} \tau^{2}>1.12\right)$ motional regimes, respectively (Fig. 1 and Fig. $S 1$ in the Supporting Information).${ }^{14}$ However, this scenery is hardly observed when increasing the MW of linear polymers, as constant $T_{1}$ and $T_{2}$ values typically arise above a fairly low MW. ${ }^{6,7}$ When modeling the dynamics of polymers two types of motions must be considered, the overall rotatory diffusion of the polymer chain as a whole (which slows down on increasing MW) and local chain motions (almost independent on MW). Indeed, for sufficiently high MW linear polymers, as the overall motion is much slower than chain local motions, it makes a negligible contribution to the effective $\tau$ ( $\tau_{\text {eff, }}$ an average of the $\tau$ for every motion affecting relaxation) and so, to $T_{1}$ and $T_{2}$ relaxation at high magnetic fields. ${ }^{6,7}$

The analysis of dendrimer dynamics by NMR relaxation has mostly relied on $T_{1}$ experiments because it is the relaxation parameter easiest to measure accurately. By analogy to linear polymers, shorter $T_{1}$ values have been associated to less mobile nuclei and hence, dendrimers with reduced local motions at the core ${ }^{10}$ or periphery ${ }^{11-}$ ${ }^{13}$ reported, attending to the topological location of the lowest $T_{1}$ values. Certainly, these simplified dynamical studies performed in the absence of additional relaxation data (e.g.; $T_{2}$, the temperature dependence of $T_{1}$, or NOE) have the advantage of a straightforward recording. However, they could lead to misinterpretations in case the relaxation behavior of dendrimers does not match with that of linear polymers because of their globular architecture.

To elucidate in detail the relaxation behaviour of dendrimers and its interpretation in terms of dynamics, Fréchet-type poly(aryl 
ether) dendrimers were selected as an illustrative example of a dendritic family where conflicting relative dynamics between core and periphery have been reported. A reduced local motion has been claimed on going from the core to the periphery of these dendrimers according to ${ }^{1} \mathrm{H} T_{1}$ relaxation, ${ }^{11,12}$ but opposite relative dynamics by alternative techniques (size exclusion chromatography with coupled molecular weight sensitive detection, ${ }^{15}$ REDOR NMR data combined with molecular modelling, ${ }^{16}$ fluorescence ${ }^{17}$ ). So, G1-G4 dendrimers shown in Fig. 1 were synthesized ${ }^{18}$ and their ${ }^{1} \mathrm{H}$ and ${ }^{13} \mathrm{C}$ NMR relaxation analyzed at various magnetic fields and temperatures $\left[T_{1}, T_{2}\right.$, selective $T_{1}$ $\left.\left(T_{1 \mathrm{~S}}\right), \mathrm{NOE}\right]$.
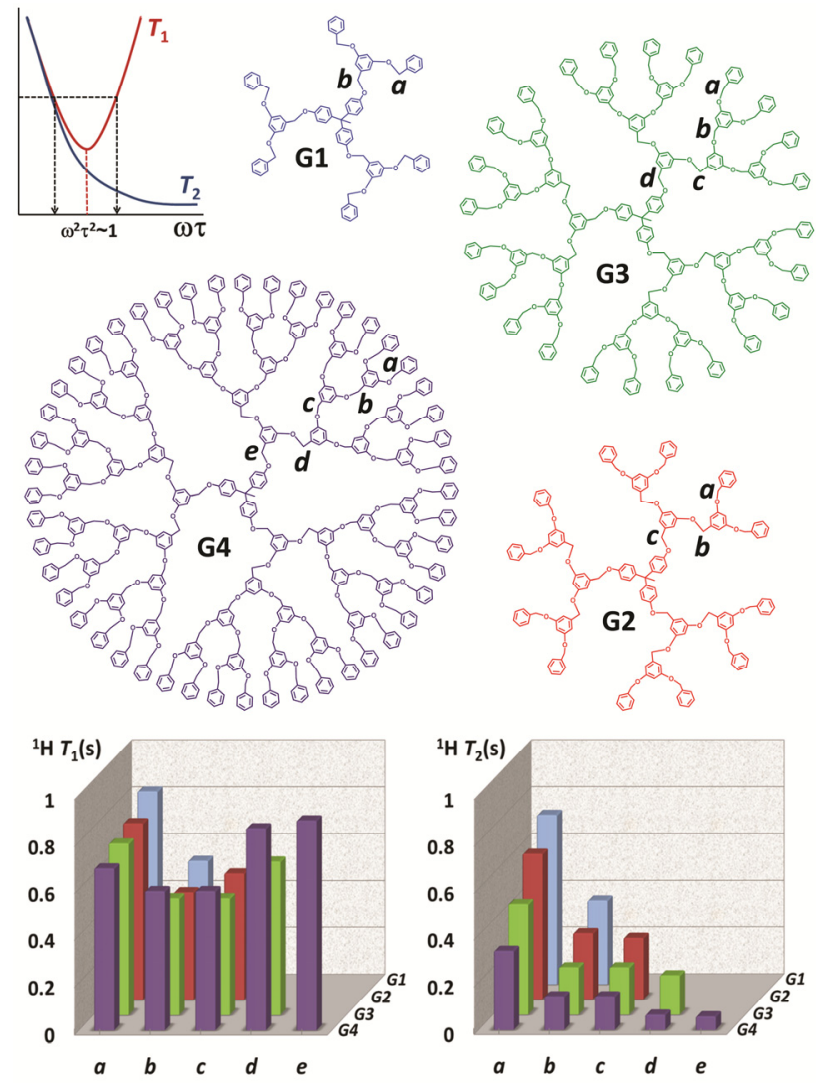

Figure 1. Structures of poly(aryl ether) G1-G4 dendrimers. Top left panel: schematic representation of the dependence of $T_{1}$ and $T_{2}$ on $\tau$. Bottom panel: ${ }^{1} \mathrm{H} T_{1}$ and $T_{2}$ for the benzylic protons of G1-G4 $\left(\mathrm{CDCl}_{3}, 500 \mathrm{MHz}, 298 \mathrm{~K}\right)$.

\section{RESULTS AND DISCUSSION}

We started measuring ${ }^{1} \mathrm{H} T_{1}$ for the benzylic protons of G3 (500 $\mathrm{MHz}, 298 \mathrm{~K}$ ) and found coincident values with those in the literature, ${ }^{11}$ with larger $T_{1}$ at the core $d \mathrm{G} 3$ and periphery $\boldsymbol{a} \mathrm{G} 3$ protons than intermediate $\boldsymbol{b G} 3$ and $\boldsymbol{c G} 3$ (Fig. 1). The analysis of G2 and G4 also revealed larger $T_{1}$ values at the core than at intermediate layers (and even periphery for G4), along with larger $T_{1}$ differences between core and intermediate protons on increasing G. Interestingly, similar ${ }^{1} \mathrm{H} T_{1}$ values resulted at the periphery independently on $\mathrm{G}$ (protons $\boldsymbol{a}$ ), but a sharp increase of $T_{1}$ was observed at the core on going from $\boldsymbol{b G} 1$ to $\boldsymbol{c G} 2, \boldsymbol{d G} 3$, and $\boldsymbol{e G} 4$. This $T_{1}$ relaxation profile agrees with that observed for other poly(aryl ether) dendrimers carrying focal units different to that shown in Fig. 1. ${ }^{11,12}$ When the dynamics of all these dendrimers has been interpreted in the light of the $T_{1}$ relaxation model of linear polymers, an increased mobility at the core of the larger dendrimer $\mathrm{G}$ (higher $T_{1}$ values) has been disclosed. ${ }^{11,12}$ To ascertain the pertinence of this dynamical analysis, a $T_{2}$ study was then carried out on G1-G4 (500 MHz, $298 \mathrm{~K}$ ), which exploits the monotonic decreases of $T_{2}$ with $\tau$. Fig. 1 shows for the benzylic protons that contrary to $T_{1}, T_{2}$ always decreases from the periphery to the core independently on $G$, pointing to a reduced mobility in the same direction. Remarkably, the variation of $T_{2}$ from G1 to G4 afforded a 53\% reduction for peripheral protons $\boldsymbol{a}$, while a much sharper $84 \%$ at the core (bG1 vs $\boldsymbol{e} \mathrm{G} 4)$, in agreement with higher $\mathrm{G}$ displaying slower dynamics and larger differences in mobility between core and periphery. Taken as a whole, these $T_{1}$ and $T_{2}$ relaxation data reveal the unsuitability of $T_{1}$ data recorded at one temperature for the analysis of dendrimer dynamics as typically done for linear polymers.

To further unravel the dynamical behaviour of G1-G4, we then investigated the temperature dependence of $T_{1}$, a parameter widely recognized as an accurate means to probe the relative dynamics within macromolecules (Fig. 2 and S2). ${ }^{7}$ The $T_{1}$ minimum with the temperature $\left(\omega^{2} \tau^{2}=1\right)$ can be used to estimate $\tau_{\text {eff, }}$ and the differences in its position as a qualitative indicator of relative rates: the lower the temperature of the $T_{1}$ minimum, the higher the rate of the motions. Fig. 2 shows the temperature dependence of $T_{1}$ for the benzylic protons of G1-G4 (500 MHz). In the case of G3, as the temperature increases, the $T_{1}$ for nuclei at intermediate layers ( $b \mathrm{G} 3$ and $c$ G3) decreases down to a minimum around $298 \mathrm{~K}$, to slightly increase afterwards. This minimum is however located at a temperature lower than $243 \mathrm{~K}$ for peripheral $\boldsymbol{a} \mathrm{G} 3$ protons, and higher than $333 \mathrm{~K}$ for core $d \mathrm{G} 3$, in agreement with an enhanced contribution of fast local motions on going from the core to the periphery. At 500 $\mathrm{MHz}$ and $298 \mathrm{~K}$, peripheral protons $\boldsymbol{a} \mathrm{G} 3$ lay at the fast motional regime, while $\boldsymbol{b} \mathrm{G} 3 / \boldsymbol{c G} 3$ close to the $T_{1}$ minimum, and core $\boldsymbol{d G} 3$ at the slow motional regime. The analysis of the temperature dependence of $T_{1}$ for G1, G2, and G4 afforded a similar prospect. It was observed that on increasing $G$, the $T_{1}$ minimum of each proton layer was shifted toward higher temperatures, in agreement with a reduction of dynamics (Fig. S4). Also, larger differences in $T_{1}$ minima were revealed between core and periphery on increasing $G$, pointing to wider distributions of local motions for dendrimers than for polymers of similar MW. Confirmation of this dynamical behaviour was obtained by studying the temperature dependence of $T_{2}$ for the benzylic protons of G1-G4 (steady increase of $T_{2}$ with the temperature; Fig. 2, S3, and S4) and of $T_{1}$ and $T_{2}$ for the aromatic protons (Fig. S5-S7). Interestingly, aromatic protons show $T_{1}$ minima shifted to lower temperatures than benzylic protons, laying at the fast motional regime at $298 \mathrm{~K}$ (with the exception of protons at the focal unit). In this scenery, a dynamical study based only on $T_{1}$ recorded at one temperature could lead to opposed motional outcomes depending on the relaxation data analysed, aromatic $v s$ benzylic, because of an interpretation pitfall of the relaxation data of the latter. 

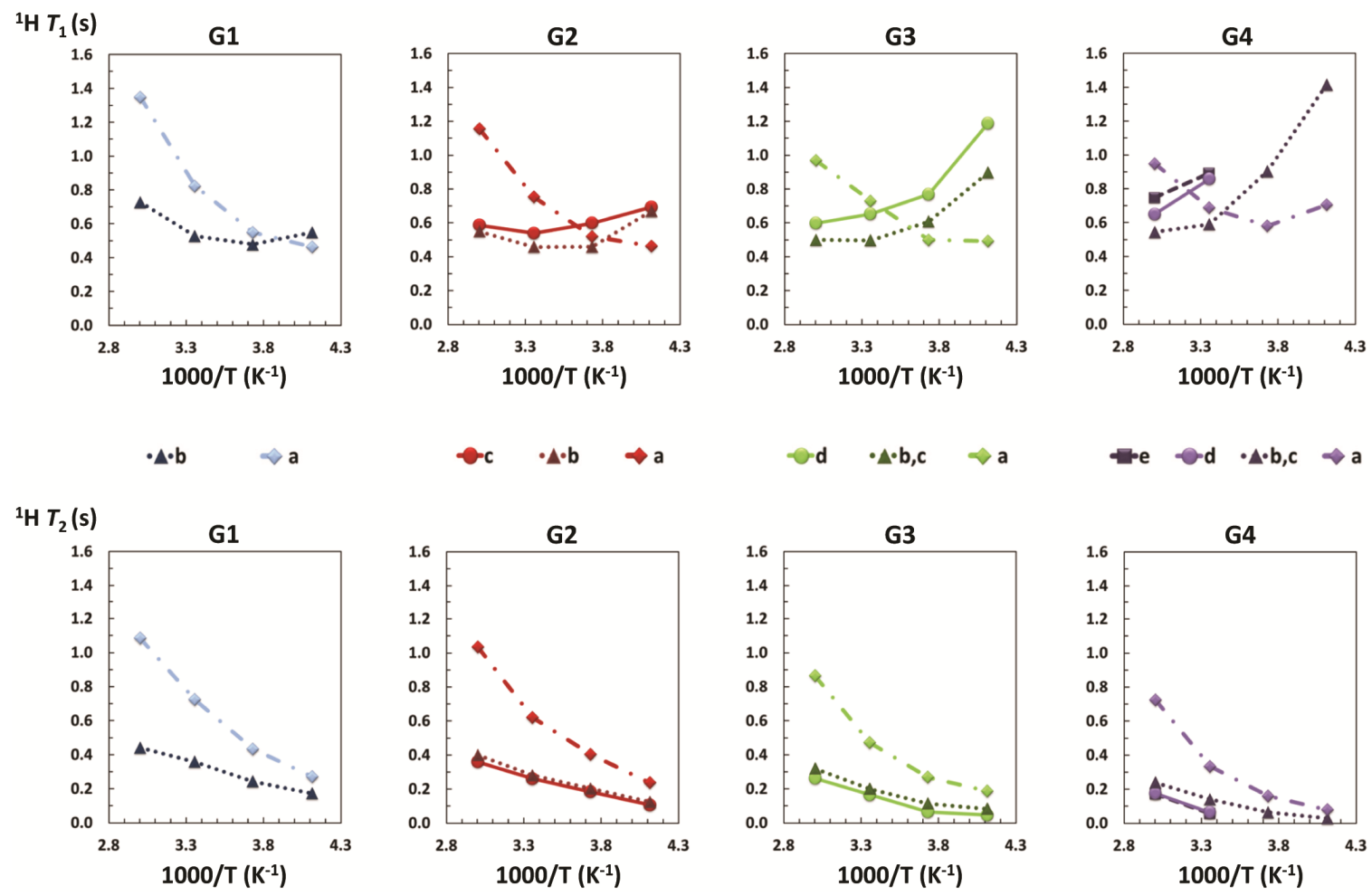

Figure 2. Temperature dependence of ${ }^{1} \mathrm{H} T_{1}$ and $T_{2}$ for the benzylic protons of G1-G4 $\left(\mathrm{CDCl}_{3}, 500 \mathrm{MHz}\right)$. Lines are guides for the eye.

Additional evidence supporting the faster dynamics of peripheral nuclei in poly(aryl ether) dendrimers was obtained from $T_{1 S}$ experiments in G3 (500 MHz, $298 \mathrm{~K}$; Supporting Information). Thus, the $T_{1 S} / T_{1}$ ratio can be used to estimate the motions involved in relaxation. ${ }^{19}$ Characteristic $T_{15} / T_{1}$ ratios close to 1 are expected for protons in the proximity of the $T_{1}$ minimum, while higher ratios up to a theoretical 1.5 for protons at $\omega^{2} \tau^{2}<<1$ and values close to zero for $\omega^{2} \tau^{2}>>1 .^{20}$ Indeed, the $T_{1 S} / T_{1}$ obtained for the wellresolved $\boldsymbol{a} \mathrm{G} 3(1.1)$ and $\boldsymbol{d G} 3(0.9)$ benzylic protons are indicative of these nuclei being at the fast and slow motional regimes, respectively, in areas close to the $T_{1}$ minima (Fig. S8).

The above dynamical picture at $500 \mathrm{MHz}$ was validated by recording ${ }^{1} \mathrm{H} T_{1}$ and $T_{2}$ at lower and higher magnetic fields. Increasing the magnetic field it is expected to produce minute enhancements in $T_{2}$, but large in $T_{1}$ especially for nuclei close to the $T_{1}$ minimum and at the slow motional regime (shift of $T_{1}$ minimum toward smaller $\left.\tau_{\text {eff }}\right)^{7,14}$ A theoretical simulation of the dependence of $T_{1}$ and $T_{2}$ with $\tau$ at three magnetic fields is shown in Fig. S1. ${ }^{1} \mathrm{H} T_{1}$ and $T_{2}$ were recorded for the benzylic and aromatic protons at 300 and $750 \mathrm{MHz}(298 \mathrm{~K})$, and compared with the data obtained at 500 $\mathrm{MHz}$ (Fig. 3 and S9). As expected, a small increase in $T_{2}$ was observed on going from 300 to $750 \mathrm{MHz}$. Large enhancements were obtained for the $T_{1}$, especially for the benzylic protons at intermediate and core layers and on increasing $G$, in agreement with these nuclei being located close to the $T_{1}$ minimum or at the slow motional regime at $298 \mathrm{~K}$ (Fig. 3). Variations in $T_{1}$ with the field were less marked for the aromatic protons as they predominantly lay at the fast motional regime at this temperature (Fig. S9). It was interesting to observe how the resonances having the lowest $T_{1}$ values moved to more peripheral layers on increasing the magnetic field (quite evident for benzylic protons in G4) as a result of the ex- pected shift of the $T_{1}$ minimum toward smaller $\tau_{\text {eff. Next, we decid- }}$ ed to study the temperature dependence of ${ }^{1} \mathrm{H} T_{1}$ and $T_{2}$ in G3 at three magnetic fields (Fig. 4 and S10). Similarly to 298 K, benzylic and aromatic protons showed almost no variation in $T_{2}$ with the magnetic field when a broader range of temperatures was analysed. As for ${ }^{1} \mathrm{H} T_{1}$, a more complicated scenery was revealed. Although increasing $T_{1}$ figures were always observed on going from 300 to $750 \mathrm{MHz}$, the temperature dependence of $T_{1}$ was strongly affected by the field and the topological location of the nuclei (periphery, intermediate layers, core). For instance, $T_{1}$ decreases for core $d \mathrm{G} 3$ proton on increasing the temperature at $750 \mathrm{MHz}$, but it remains rather unaffected at $300 \mathrm{MHz}$. On the contrary, peripheral $\boldsymbol{a} \mathrm{G} 3$ always shows increasing $T_{1}$ values with the temperature independently on the field, although larger enhancements resulted at $300 \mathrm{MHz}$. In addition, converging $T_{1}$ values were always seen for the three fields on increasing the temperature. A detailed analysis of these variations of $T_{1}$ for benzylic and aromatic protons, bearing in mind the theoretical dependence of $T_{1}$ with $\tau$ and the magnetic field (Fig. S1), has allowed us to draw a clear picture of the dynamics of these dendrimers. As schematically represented in Fig. 4 for the benzylic protons of $\mathrm{G} 3$, on going from the periphery to the core, nuclei in dendrimers experience a reduced mobility characterized by larger $\tau_{\text {eff. }}$ On increasing the dendrimer $G$, additional nuclei with even larger $\tau_{\text {eff }}$ are added at the core which results in a progressively wider distribution of local motions. Since the translation of $\tau_{\text {eff }}$ into $T_{1}$ values depends on the magnetic field and the nuclei's motional regime, only the recording of $T_{1}$ data at various temperatures can ensure the correct description of dendrimer dynamics. Alternatively, a detailed dynamical analysis can be obtained by recording $T_{2}$ at one temperature, although no information on the nuclei's motional regime is gained in this way. 

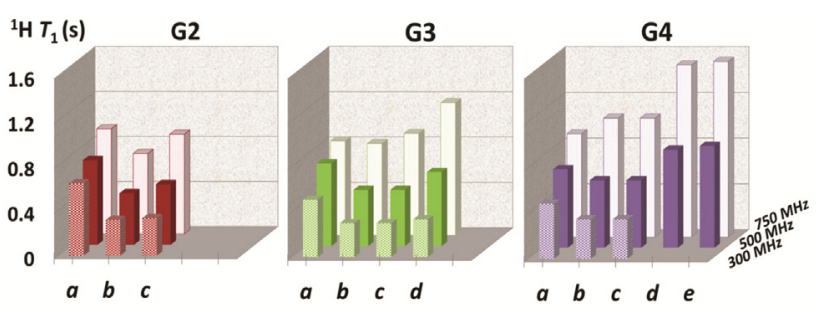

${ }^{1} \mathrm{H} T_{2}(s)$

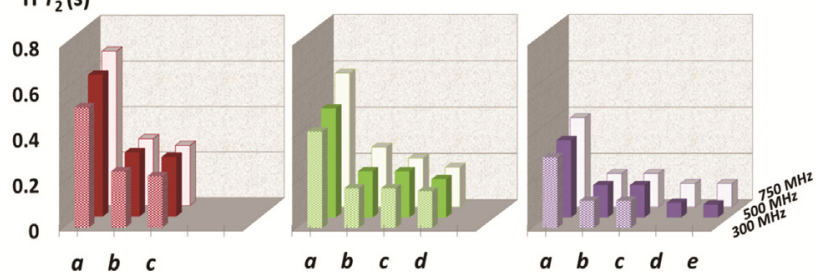

Figure 3. ${ }^{1} \mathrm{H} T_{1}$ and $T_{2}$ values for the benzylic protons of G2-G4 as a function of the magnetic field $\left(\mathrm{CDCl}_{3}, 298 \mathrm{~K}\right)$.
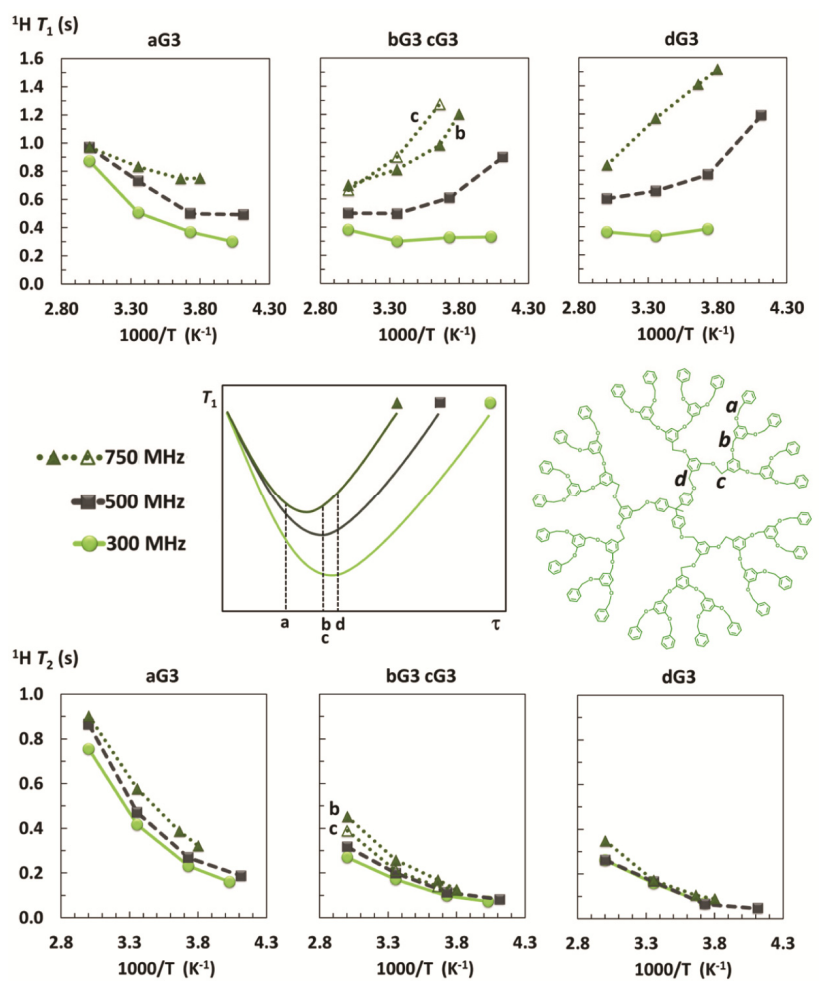

Figure 4. Top and bottom panels: temperature dependence of ${ }^{1} \mathrm{H} T_{1}$ and $T_{2}$ for the benzylic protons of G3 at 300,500 , and $750 \mathrm{MHz}$ $\left(\mathrm{CDCl}_{3}\right)$. Middle panel: schematic representation of the $T_{1}$ curves for the benzylic protons of $\mathrm{G} 3(\boldsymbol{a}, \boldsymbol{b}, \boldsymbol{c}, \boldsymbol{d})$ at the three magnetic fields and their respective locations at $298 \mathrm{~K}$.

Dynamical studies by ${ }^{1} \mathrm{H}$ relaxation benefit from a fast data acquisition due to the high natural abundance of the ${ }^{1} \mathrm{H}$ nucleus and its large gyromagnetic ratio. Conversely, ${ }^{1} \mathrm{H}$ relaxation in large macromolecules might suffer from spin diffusion, an extensive crossrelaxation phenomenon efficient in the slow motional regime.
Whereas dipolar relaxation diminishes with the sixth power of the distance, leading to substantial magnetization being transferred only to the most nearby protons, spin diffusion rapidly propagates through the network of protons to utterly affect nuclei located quite apart. Consequently, spin diffusion leads to $T_{1}, T_{1 S}$, and $T_{2}$ data loosing most of their local dynamical details. ${ }^{714}$ Under these circumstances, ${ }^{13} \mathrm{C}$ relaxation studies are recommended as they are dominated by dipolar interactions with directly bonded protons, ensuring the efficient probe of local dynamics. ${ }^{7}$

Although spin diffusion can be anticipated as negligible in the poly(aryl ether) dendrimers herein analyzed [i) molecular weight range; ii) $T_{1 S} / T_{1}$ ratios close to 1 ; iii) integration of cross peaks in ${ }^{1} \mathrm{H}-{ }^{1} \mathrm{H}$ NOESY for $\mathrm{G} 3$ account for only $1-2 \%$ of the corresponding diagonal peak intensity (mix. time $350 \mathrm{~ms}, 298 \mathrm{~K}$, Supporting Information)], we decided to perform a ${ }^{13} \mathrm{C}$ relaxation study $\left(T_{1}, T_{2}\right.$, heteronuclear $\left.{ }^{13} \mathrm{C}\left\{{ }^{1} \mathrm{H}\right\} \mathrm{NOE}\right)$ at various fields $(125$ and $188 \mathrm{MHz})$ and temperatures. With the aim of increasing the sensitivity of the usually long ${ }^{13} \mathrm{C}$ experiments, we have taken advantage of indirect detected experiments based on the HSQC sequence. ${ }^{21}$ This way, not only faster $T_{1}, T_{2}$, and NOE experiments were recorded, but strong signal overlapping in the ${ }^{13} \mathrm{C}$ spectra was surpassed. Moreover, by reducing the dimensionality of the experiments to the more resolved ${ }^{1} \mathrm{H}$ 1D dimension, even larger savings in spectrometer time resulted in addition to a simplified signal integration task.
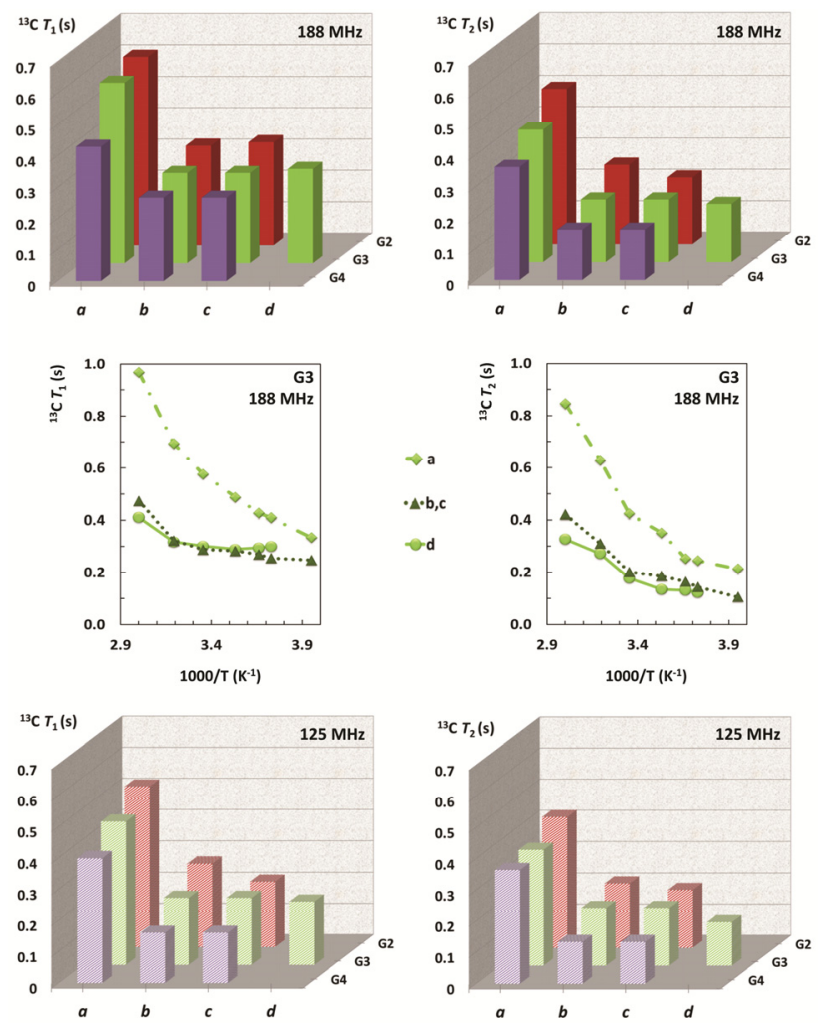

Figure 5. Top and bottom panels: ${ }^{13} \mathrm{C} T_{1}$ and $T_{2}$ for the benzylic carbons of G2-G4 at 188 and $125 \mathrm{MHz}\left(\mathrm{CDCl}_{3}, 298 \mathrm{~K}\right)$. Middle panel: Temperature dependence of ${ }^{13} \mathrm{C} T_{1}$ and $T_{2}$ for the benzylic carbons of $\mathrm{G} 3\left(\mathrm{CDCl}_{3}, 188 \mathrm{MHz}\right)$. Lines are guides for the eye. 

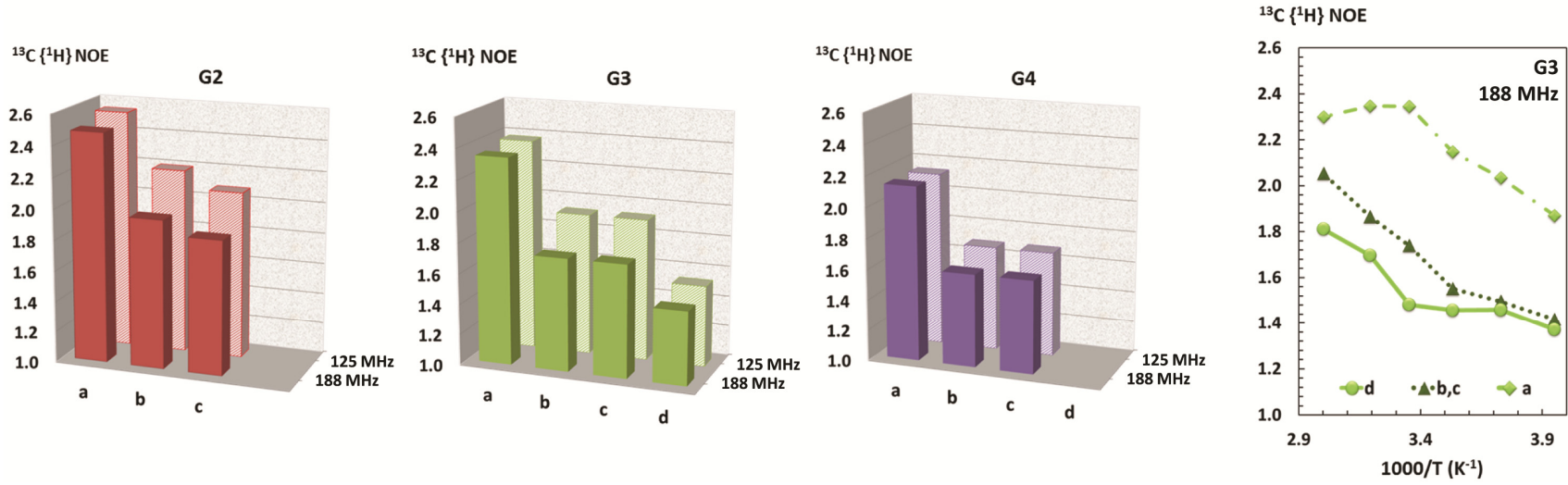

Figure 6. ${ }^{13} \mathrm{C}\left\{{ }^{1} \mathrm{H}\right\} \mathrm{NOE}$ for the benzylic C-H of G2-G4 at 125 and $188 \mathrm{MHz}\left(\mathrm{CDCl}_{3}, 298 \mathrm{~K}\right)$. Right panel: Temperature dependence of ${ }^{13} \mathrm{C}\left\{{ }^{1} \mathrm{H}\right\} \mathrm{NOE}$ for the benzylic $\mathrm{C}-\mathrm{H}$ of $\mathrm{G} 3\left(\mathrm{CDCl}_{3}, 188 \mathrm{MHz}\right)$. Lines are guides for the eye.

Gratifyingly, this ${ }^{13} \mathrm{C}$ study confirmed the validity of the relative dynamics extracted from ${ }^{1} \mathrm{H}$ relaxation. As seen in Fig. 5 and $\mathrm{S} 11$, benzylic and aromatic carbons in G2-G4 displayed $T_{1}$ and $T_{2}$ values which confirmed the reduction of mobility on going from the periphery to the core and at higher $\mathrm{G}$ (higher $T_{1}$ than $T_{2}$; lower $T_{2}$ at internal layers and on increasing G; $T_{1}$ follows a similar trend to $T_{2}$ although with slightly increasing values being observed at the more internal layers). In addition, $T_{1}$ and $T_{2}$ increased with the magnetic field as expected, showing larger enhancements for $T_{1}$ than $T_{2}$. The variations of $T_{1}$ and $T_{2}$ with the temperature were also studied for G3 (Fig. 5 and S12), showing complete consistency with the aforementioned dynamical analysis (reduction of $T_{2}$ on lowering the temperature and $T_{1}$ showing the expected minima for the more internal carbons: $d \mathrm{G} 3, \mathrm{CG} 3, \mathrm{ZG} 3$ ).

Similar conclusions were also obtained by studying the ${ }^{13} \mathrm{C}\left\{{ }^{1} \mathrm{H}\right\}$ NOE for G2-G4 at two magnetic fields. Theoretically, for a ${ }^{13} \mathrm{C}-{ }^{1} \mathrm{H}$ pair, the ${ }^{13} \mathrm{C}\left\{{ }^{1} \mathrm{H}\right\}$ NOE varies with $\tau$ between 2.988 for $\omega^{2} \tau^{2}<<1$ and 1.15 for $\omega^{2} \tau^{2}>>1$. In between these limiting regimes, ${ }^{13} \mathrm{C}\left\{{ }^{1} \mathrm{H}\right\} \mathrm{NOE}$ is expected to increase with the temperature and decrease with the magnetic field. Indeed, a ${ }^{13} \mathrm{C}\left\{{ }^{1} \mathrm{H}\right\} \mathrm{NOE}$ analysis for the benzylic and aromatic carbons in G2-G4 afforded decreasing NOE values from the periphery to the core and on increasing $\mathrm{G}$, in agreement with a reduction of dynamics and the adoption of larger $\tau_{\text {eff }}$ (Fig. 6 and S13). In addition, lower NOE values were obtained on increasing the magnetic field. When the temperature dependence of ${ }^{13} \mathrm{C}\left\{{ }^{1} \mathrm{H}\right\}$ NOE was studied in $\mathrm{G} 3$, decreasing values were seen for benzylic and aromatic $\mathrm{C}-\mathrm{H}$ on lowering the temperature, in harmony with the expected reduction of dynamics.

The dynamical framework herein presented can be interpreted in the light of recent theoretical studies on the relaxation spectra of dendrimers by Markelov and coworkers. ${ }^{22}$ These authors have proposed the dendrimer segmental orientational mobility as governed by three main relaxation processes with rather different characteristic times: (i) the global rotation of the dendrimer as a whole [rotational correlation time $\left(\tau^{\mathrm{rot}}\right)$, which increases with G], (ii) the turns of a dendrimer sub-branch originating from a given segment [pulsating correlation time $\left(\tau^{\text {pul }}\right)$, which is independent on $\mathrm{G}$ and in- creases with the topological distance between that segment and the dendrimer periphery], and (iii) the local reorientation of individual segments [internal correlation time $\left(\tau^{\text {int }}\right)$, practically independent on $\mathrm{G}$ and the segment topological location]. Indeed, the slower dynamics observed on going from the periphery to the core in G1$\mathrm{G} 4$ respond to the proposed increase of $\tau^{\text {pul }}$. At the same time, at higher $G$, not only $\tau^{\text {rot }}$ increases, but also new internal layers appear characterized by even larger $\tau^{\text {pul }}$, which results in wider distributions of local motions than linear polymers of similar MW. It is also interesting to stress that in contrast to linear polymers, the globular architecture of dendrimers turns into overall motions fast enough to significantly contribute to relaxation.

\section{CONCLUSIONS}

We have shown that the evaluation of dendrimer dynamics only on the basis of ${ }^{1} \mathrm{H}$ or ${ }^{13} \mathrm{C} T_{1}$ relaxation data at one temperature can lead to misinterpretations. Dendrimers present slow internal dynamics and hence, nuclei may reside in the slow motional regime and display increasing $T_{1}$ values on going from the periphery to the core and at higher $\mathrm{G}$ (larger $\tau_{\text {eff }}$ ). In contrast to the relaxation properties typically observed for linear polymers, these $T_{1}$ increments should not be interpreted as resulting from faster dynamics. Since an accurate analysis of $T_{1}$ depends on the magnetic field and nuclei's motional regime, only the recording of $T_{1}$ data at various temperatures (or fields) ensures the correct description of dendrimer dynamics. Fast and reliable information on the motions involved in the relaxation of dendrimers can be also obtained by determining $T_{2}$ or ${ }^{13} \mathrm{C}\left\{{ }^{1} \mathrm{H}\right\} \mathrm{NOE}$ data at one temperature, however, no information on the nuclei's motional regime is gained in this way. The large number of dendritic families, other than poly(aryl ether), where dynamics have been evaluated on the basis of $T_{1}$ data at one temperature $^{10,13}$ unveils the relevance of these results and urges necessity of revisiting previous studies. With the aim of further unravelling the dynamics of dendrimers, quantitative NMR analysis are much awaited implementing spectral density functions that weight the influence of overall and local motions as a function of $G$ and the nuclei's topological location. 


\section{ASSOCIATED CONTENT}

Supporting Information. NMR methods, relaxation data, dendrimer synthesis and characterization. This material is available free of charge via the Internet at http://pubs.acs.org.

\section{AUTHOR INFORMATION}

\section{Corresponding Author}

ricardo.riguera@usc.es; ef.megia@usc.es

\section{ACKNOWLEDGMENT}

This work was financially supported by the Spanish Government (CTQ2009-10963 and CTQ2009-14146-C02-02) and the Xunta de Galicia (10CSA209021PR and CN2011/037). Luiz F. Pinto thanks the Portuguese Foundation for Science and Technology (FCT MCTES) for a Ph.D. grant (SFRH/BD/37341/2007).

\section{REFERENCES}

(1) (a) Caminade, A.-M.; Turrin, C.-O.; Laurent, R.; Ouali, A.; Delavaux-Nicot, B. Dendrimers: Towards Catalytic, Material and Biomedical Uses; John Wiley \& Sons, Ltd: Chichester, UK., 2011. (b) Astruc, D.; Boisselier, E.; Ornelas, C. Chem. Rev. 2010, 110, 1857. (c) Menjoge, A. R.; Kannan, R. M.; Tomalia, D. A. Drug Discov. Today 2010, 15, 171. (d) Vögtle, F.; Richardt, G.; Werner, N. Dendrimer Chemistry; Wiley-VCH: Weinheim, 2009. (e) Lee, C. C.; MacKay, J. A.; Fréchet, J. M. J.; Szoka, F. C. Nat. Biotechnol. 2005, 23, 1517.

(2) de Gennes, P. G.; Hervet, H. J. Physique Lett. 1983, 44, 351.

(3) Lescanec, R. L.; Muthukumar, M. Macromolecules 1990, 23, 2280.

(4) Ballauff, M.; Likos, C. N. Angew. Chem., Int. Ed. 2004, 43, 2998.

(5) Palmer III, A. G. Chem. Rev. 2004, 104, 3623.

(6) Heatley, F. Progr. Nucl. Magn. Reson. Spectrosc. 1979, 13, 47.

(7) Dais, P.; Spyros, A. Progr. Nucl. Magn. Reson. Spectrosc. 1995, 27, 555.

(8) (a) Novoa-Carballal, R.; Säwén, E.; Fernandez-Megia, E.; Correa, J.; Riguera, R.; Widmalm, G. Phys. Chem. Chem. Phys. 2010, 12, 6587. (b) Meltzer, A. D.; Tirrell, D. A.; Jones, A. A.; Inglefield, P. T.; Hedstrand, D. M.; Tomalia, D. A. Macromolecules 1992, 25, 4541. (c) For a quantitative ${ }^{2} \mathrm{H}$ relaxation study on PAMAM dendrimers, see: Meltzer, A. D.; Tirrell, D. A.; Jones, A. A.; Inglefield, P. T. Macromolecules 1992, 25, 4549.

(9) Reports describing increased local motions at the periphery of dendrimers based on various relaxation parameters: (a) Moreno, K. X.; Simanek, E. E. Macromolecules 2008, 41, 4108. (b) Fernandez-Megia, E.; Correa, J.; Riguera, R. Biomacromolecules 2006, 7, 3104. (c) Baille, W. E.; Malveau, C.; Zhu, X. X.; Kim, Y. H.; Ford, W. T. Macromolecules 2003, 36, 839. (d) Malveau, C.; Baille, W. E.; Zhu, X. X.; Ford, W. T. J. Polym. Sci., Part B: Polym. Phys. 2003, 41, 2969. (e) Chai, M.; Niu, Y.; Youngs, W. J.; Rinaldi, P. L. J. Am. Chem. Soc. 2001, 123, 4670. (f) Gorman, C. B.; Hager, M. W.; Parkhurst, B. L.; Smith, J. C. Macromolecules 1998, 31, 815.

(10) Reports describing increased local motions at the periphery of dendrimers based on $T_{1}$ only: (a) Welch, K. T.; Arévalo, S.; Turner, J. F. C.; Gómez, R. Chem. Eur. J. 2005, 11, 1217. (b) Zloh, M.; Ramaswamy, C.; Sakthivel, T.; Wilderspin, A.; Florence, A. T. Magn. Reson. Chem. 2005, 43, 47.

(11) Hecht, S.; Fréchet, J. M. J. J. Am. Chem. Soc. 1999, 121, 4084.

(12) $T_{1}$ relaxation studies describing increased local motions at the core of poly(aryl ether) dendrimers: (a) Kimata, S.-I.; Jiang, D.-L.; Aida, T. J.
Polym. Sci., Part A: Polym. Chem. 2003, 41, 3524. (b) Jiang, D.-L.; Aida, T. J. Am. Chem. Soc. 1998, 120, 10895. (c) Jiang, D.-L.; Aida, T. Nature 1997, 388, 454. (d) Tomoyose, Y.; Jiang, D. L.; Jin, R. H.; Aida, T.; Yamashita, T.; Horie, K.; Yashima, E.; Okamoto, Y. Macromolecules 1996, 29, 5236.

(13) $T_{1}$ relaxation studies describing increased local motions at the core of non-poly(aryl ether) dendrimers: (a) Uyemura, M.; Aida, T. Chem. Eur. J. 2003, 9, 3492. (b) Buschhaus, B.; Bauer, W.; Hirsch, A. Tetrahedron 2003, 59, 3899. (c) van Manen, H. J.; Fokkens, R. H.; Nibbering, N. M. M.; van Veggel, F. C. J. M.; Reinhoudt, D. N. J. Org. Chem. 2001, 66, 4643.

(14) Kowalewski, J.; Mäler, L. Nuclear Spin Relaxation in Liquids: Theory, Experiments, and Applications; CRC Press: Boca Raton, 2006.

(15) Mourey, T. H.; Turner, S. R.; Rubinstein, M.; Fréchet, J. M. J.; Hawker, C. J.; Wooley, K. L. Macromolecules 1992, 25, 2401.

(16) (a) Kao, H.-M.; Stefanescu, A. D.; Wooley, K. L.; Schaefer, J. Macromolecules 2000, 33, 6214. (b) Wooley, K. L.; Klug, C. A.; Tasaki, K.; Schaefer, J. J. Am. Chem. Soc. 1997, 119, 53.

(17) (a) Li, Y.-Y.; Han, L.; Chen, J.; Zheng, S.; Zen, Y.; Li, Y.; Li, S.; Yang, G. Macromolecules 2007, 40, 9384. (b) De Backer, S.; Prinzie, Y.; Verheijen, W.; Smet, M.; Desmedt, K.; Dehaen, W.; De Schryver, F. C. J. Phys. Chem. A 1998, 102, 5451.

(18) (a) Yamazaki, N.; Washio, I.; Shibasaki, Y.; Ueda, M. Org. Lett. 2006, 8, 2321. (b) Hawker, C. J.; Fréchet, J. M. J. J. Am. Chem. Soc. 1990 112,7638 .

(19) Niccolai, N.; Garsky, V.; Gibbons, W. A. J. Am. Chem. Soc. 1980, $102,1517$.

(20) Freeman, R.; Hill, H. D. W.; Tomlinson, B. L.; Hall, L. D. J. Chem. Phys. 1974, 61, 4466.

(21) Farrow, N. A.; Muhandiram, R.; Singer, A. U.; Pascal, S. M.; Kay, C. M.; Gish, G.; Shoelson, S. E.; Pawson, T.; Forman-Kay, J. D.; Kay, L. E. Biochemistry 1994, 33, 5984 .

(22) Markelov, D. A.; Lyulin, S. V.; Gotlib, Y. Y.; Lyulin, A. V.; Matveev, V. V.; Lahderanta, E.; Darinskii, A. A. J. Chem. Phys. 2009, 130, 044907.

Table of Contents artwork

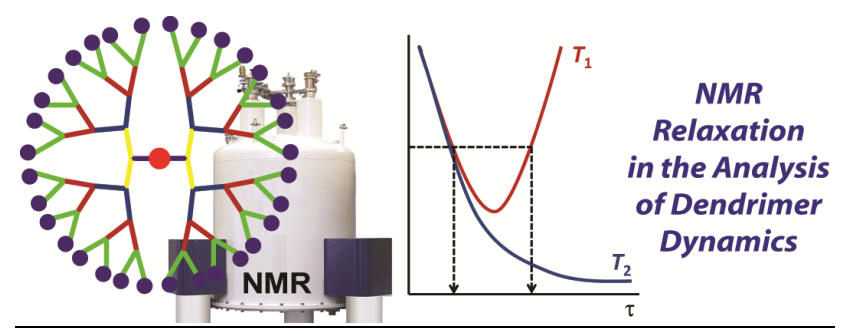

